\title{
Equipamentos de Proteção Individual Impressos em 3D por Instituições de Ensino Federais para - Enfrentamento da COVID-19
}

\author{
Personal Protective Equipment Printed in 3D by Federal Education \\ Institutions to Confront COVID-19
}

Amanda Ferreira dos Santos ${ }^{1}$

Iulas de Souza Ramos ${ }^{1}$

Ana Clara Silva dos Santos ${ }^{1}$

Grasiely Faccin Borges ${ }^{1}$

Ita de Oliveira e Silva ${ }^{1}$

Jane Mary de Medeiros Guimarães ${ }^{1}$

${ }^{1}$ Universidade Federal do Sul da Bahia, Itabuna, BA, Brasil

\begin{abstract}
Resumo
A pandemia causada pelo coronavírus (SARS-CoV-2), associada à escassez dos Equipamentos de Proteção Individuais (EPIs), apresenta-se como uma ameaça à saúde global. O objetivo do estudo foi identificar a produção de EPIs impressos em 3D nas Instituições de Ensino Federais para o enfrentamento da COVID-19. A fonte de pesquisa foi o banco de dados do Portal do Ministério da Educação de Monitoramento nas Instituições de Ensino. Até o momento, 89 instituições estavam produzindo três modelos diferentes de EPIs em 3D. Os modelos encontrados foram os Protetores Faciais tipo "Face Shield", as máscaras similares ao modelo N-95 e a máscara com uma cúpula em formato semicilíndrico. As Instituições de Ensino Federais estão colocando a tecnologia e a ciência à disposição da sociedade brasileira no combate à COVID-19, buscando responder à crise global com a impressão 3D, de forma segura, com inovação, baixo custo e com rápida produção.
\end{abstract}

Palavras-chave: Equipamento de Proteção Individuais. Impressão 3D. Instituições de Ensino Federais.

\begin{abstract}
A coronavirus pandemic (SARS-CoV-2), associated with a shortage of Personal Protective Equipment (PPEs) presents itself as a threat to global health. The objective of the study was to identify a production of PPE printed in 3D, in Federal Education Institutions in the confrontation of COVID-19. The research source of the Ministry of Education's Monitoring database in the Teaching Institutions. To date, 89 institutions were producing three different 3D PPE models. The models found were "Face Shield" face protectors, models of masks similar to N-95 and a dome in a semi-cylindrical shape. Federal Education Institutions are making technology and science available to Brazilian society in the fight against COVID-19, seeking to respond to the global crisis with 3D printing, safely, with innovation, low cost and rapid production.
\end{abstract}

Keywords: Personal Protective Equipment. 3D Printing. Federal Education Institutions.

Área Tecnológica: Tecnologia. Gestão. Saúde Pública. 


\section{Introdução}

A COVID-19 (sigla do inglês para Corona Virus Disease) passou a ser considerada pela Organização Mundial da Saúde (OMS) uma pandemia em março de 2020 (WHO, 2020). Causada pelo coronavírus (SARS-CoV-2), essa doença apresenta-se como uma potencial ameaça à saúde global, seja pela sua capacidade de sobrecarregar os sistemas de saúde em todo o mundo, seja pelo fato de gerar elevadas taxas de mortalidade.

Um dos problemas que emergiu com a pandemia, foi a falta de materiais no enfrentamento da COVID-19, principalmente no que tange aos Equipamentos de Proteção Individuais (EPIs). Esse fator está estritamente atrelado a um cenário de incertezas sobre o patógeno viral, sua viabilidade no ambiente e a transmissibilidade das partículas infectantes, das quais são expressas tipicamente pelo contato com mucosas e com sua persistência sobre superfícies inanimadas, como metal, vidro ou plástico (KAMPF et al., 2020; VAN DOREMALEN et al., 2020).

De acordo com a Norma Regulamentadora n. 6 (NR-6), da Portaria n. 3.214/78 MTB, considera-se EPI todo dispositivo ou produto de uso individual utilizado pelo trabalhador destinado à proteção aos riscos suscetíveis de ameaçar a segurança e a saúde no trabalho (BRASIL, 1978). Esses equipamentos são indispensáveis para os profissionais de saúde e, no contexto pandêmico, são essenciais para a proteção dos trabalhadores e para o impedimento da transmissão no ambiente de trabalho, bem como para a gestão da pandemia, uma vez que trabalhadores da saúde infectados também mediam contágios.

As tecnologias e recursos existentes não estão sendo suficientes para os pacientes e profissionais da área da saúde (RAMSEY, 2020; OPAS, 2020). Relatos de contaminação e até mesmo de mortes de profissionais infectados trazem à tona evidências da estrita necessidade da adoção com rigor das medidas de proteção individuais (MOOCK; MELLO, 2020). Mesmo em locais como Japão, China, Índia, Coreia do Sul, Austrália e Irã, países em que os protocolos de assistência já estão bem definidos e não há restrição de acesso aos equipamentos de segurança, ainda é elevada a incidência do contágio e de morte entre os profissionais de saúde (TANNE et al., 2020).

O Conselho Federal de Medicina (CFM) e o Conselho Federal de Enfermagem (COFEN) têm cobrado rigor na utilização dos EPIs, os Conselhos têm também acolhido denúncias sobre os locais onde faltam esses equipamentos. O número de relatos e de denúncias aumentou e, consequentemente, também houve elevação do número de profissionais infectados, refletindo o avanço da pandemia. Sindicatos municipais e conselhos dessas categorias continuam a contabilizar um alto número de infectados, e a Associação Nacional de Medicina do Trabalho já expôs sua preocupação diante o histórico dos profissionais de saúde infectados e mortos no exterior e os alarmantes números no Brasil. Até abril de 2020, cerca de 4 mil profissionais estavam afastados pela doença, sendo 552 com diagnóstico confirmado e mais de 3,5 mil em investigação. De acordo com o Cofen, foram contabilizadas mais de 4,8 mil denúncias por falta de EPIs para o trabalho no enfrentamento à COVID-19 (ANAMT, 2020).

Em resposta à crise de saúde global, houve o desenvolvimento e a disseminação generalizada de tecnologias de manufatura distribuída em massa. Projetos foram desenvolvidos, compartilhados e replicados em diferentes demandas e escalas. Entre essas tecnologias, destaca-se o uso da impressora 3D, também conhecida como prototipagem rápida e que apresenta um enorme potencial para mudanças revolucionárias dentro da área da saúde (MATOZINHOS et al., 2017). 
Em resposta ao atual cenário, a comunidade que dispõe e desenvolve trabalhos com a impressão 3D está oferecendo ferramentas e mecanismos para produzir rapidamente equipamentos para auxiliar os profissionais no combate à COVID-19 (PRUSA PRINTERS, 2020). Na luta contra a COVID-19, pode-se destacar as Instituições de Ensino Superior públicas brasileiras, que estão realizando seu papel social de levar ensino, pesquisa, extensão e desenvolvimento para toda a sociedade local e global, aplicando o conhecimento, as tecnologias e as ciências desenvolvidas na busca de resoluções para a problemática atual (BRASIL, 2020a).

Modelos de produção colaborativa de máscaras para proteção individual com base no processo de fabricação digital e de cultura maker têm sido documentados no período de pandemia (JORGE et al., 2020; PROJETO HÍGIA, 2020). No entanto, ainda são necessários estudos que reúnam essas informações e que possam dar suporte para auxiliar projetos futuros, tendo em vista a escassez dos recursos em meio a um cenário nacional, que é caracterizado pela diversidade e pela desigualdade do tecido social, concomitante ao crescimento de casos, à transmissão sustentada e à escassez de EPIs. Desse modo, este trabalho tem como objetivo fazer um levantamento sobre os projetos brasileiros existentes na comunidade acadêmica sobre a produção de EPIs impressos em 3D no combate à pandemia da COVID-19, de modo a vislumbrar as inúmeras possibilidades de ações na prevenção e no tratamento do coronavírus.

\section{Metodologia}

Trata-se de uma pesquisa descritiva, exploratória e quantitativa. $\mathrm{O}$ trabalho foi realizado por meio de diferentes etapas. A primeira etapa foi realizada mediante pesquisa na base de dados do Portal do Ministério da Educação de Monitoramento nas Instituições de Ensino (http:// portal.mec.gov.br/coronavirus/).

No banco de dados do Ministério da Educação, foram selecionadas instituições de Ensino Superior que estão produzindo EPIs para o combate à pandemia. Foram excluídos os estudos das Instituições que não citavam a produção de EPIs impressos em 3D como atividade.

Em seguida, foram acessados os sites de cada universidade listada na etapa anterior. Para a busca de informações do estudo, foram utilizados os descritores em ciências da Saúde (DeCS): "Equipamentos de Proteção individual"; "EPIs com impressora 3D"; "Impressora 3D no combate à COVID-19". Os descritores foram cruzados simultaneamente por meio do operador booleano "AND".

O período de seleção e de coleta das informações ocorreu até o dia 12 de maio de 2020. Após esse período, iniciou-se a coleta de dados, na qual foi aplicado um formulário padronizado em busca das seguintes informações de cada estudo: instituição, cidade, estado, coordenadores, título/modelo desenvolvido, tipo de financiamento. Posteriormente, três pesquisadores tabularam e sintetizaram as informações.

Na última etapa do estudo, foi realizado um mapeamento a partir de uma sistematização quantitativa por regiões do Brasil (Norte, Nordeste, Centro-Oeste, Sul, Sudeste), apresentando o número de instituições de cada região e as siglas das instituições que estão produzindo. Os resultados foram apresentados de forma descritiva. Também foram utilizadas imagens dos resultados e dos modelos desenvolvidos para melhor visualização. 


\section{Resultados e Discussão}

De acordo com os dados encontrados por meio das buscas no Portal Coronavírus - Monitoramento nas Instituições de Ensino, pertencente ao Ministério da Educação, foram contabilizados 89 registros de Institutos e Universidades Federais que realizavam a produção de EPIs com impressoras 3D. As instituições estão distribuídas em todas as cinco regiões do país, sendo 13 instituições na Região Norte, 23 na Região Nordeste, 13 na Região Centro-Oeste, 23 na Região Sudeste e 16 na Região Sul. Todas empenhadas em ajudar seu território no combate ao novo coronavírus (Figura 1).

Figura 1 - Instituições de Ensino Federal no Brasil que produziram Protetores Faciais com impressora 3D

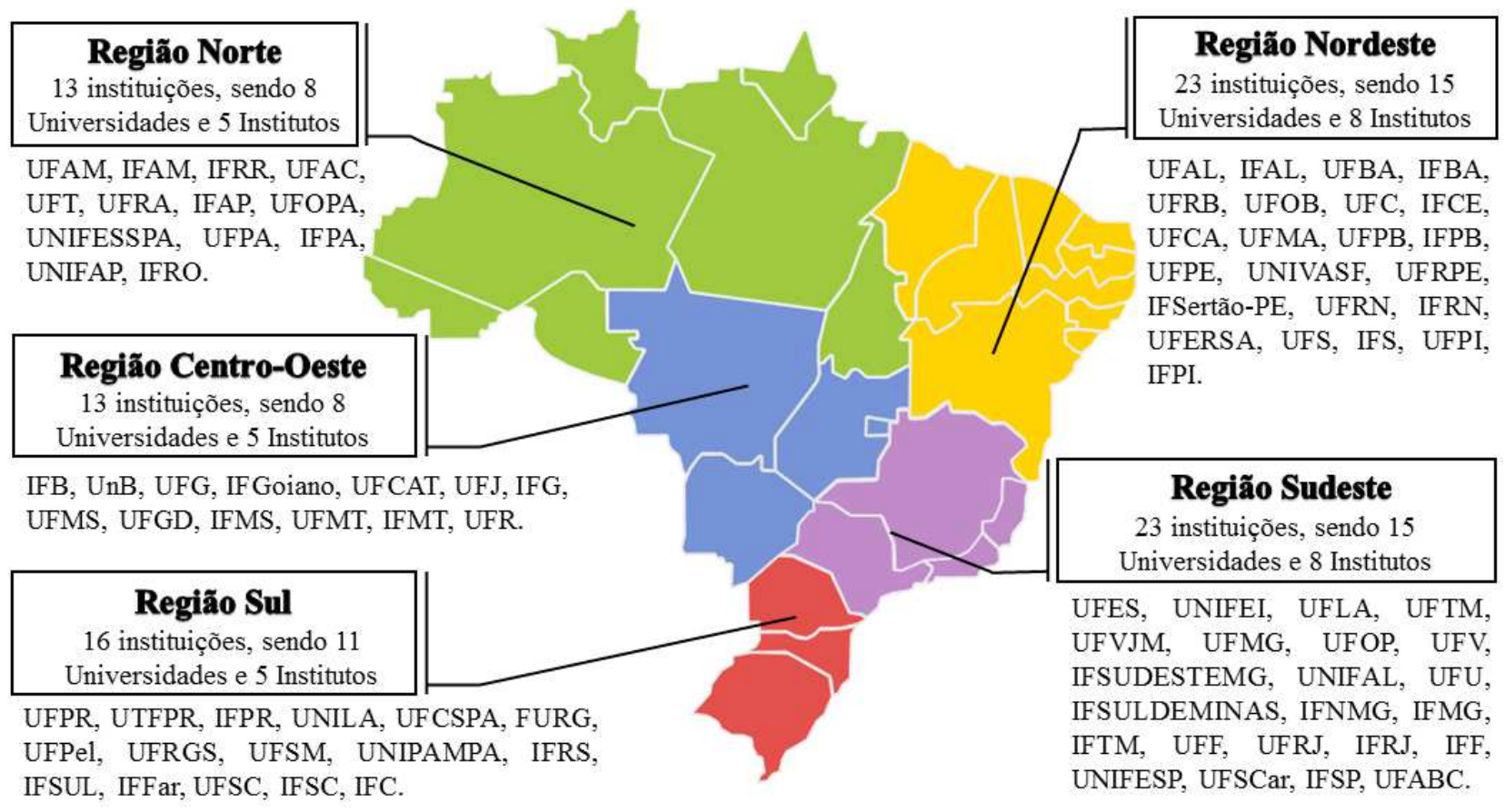

Fonte: Elaborada pelos autores deste artigo (2020)

Estão sendo produzidos três modelos diferentes de EPIs em 3D pelas instituições pesquisadas. Os modelos encontrados foram os Protetores Faciais tipo "Face Shield", as máscaras similares ao modelo N-95 e a máscara com uma cúpula em formato semicilíndrico (Figura 2). Todas as produções foram pensadas para prevenção, pois diante da forma de contaminação desse vírus, todo profissional de saúde que atender os pacientes com suspeita de Síndrome Gripal deve usar EPIs e adotar as medidas para evitar contágio (BRASIL, 2020b; JONES, 2020), tendo em vista que, a depender dos procedimentos realizados, podem ser geradas gotículas, secreções e aerossóis, com isso, ocasionando diferentes graus de risco de infecção. A exemplo, é possível citar as manobras de vias aéreas que são consideradas de alto risco e indispensáveis na terapia da COVID-19 (LOCKHART et al., 2020). 
Figura 2 - Modelos de protótipos impressos em 3D: (A) Protótipo de protetores faciais; (B) Protótipo de máscara similar ao modelo N95; (C) Protótipo da cúpula em formato semicilíndrico

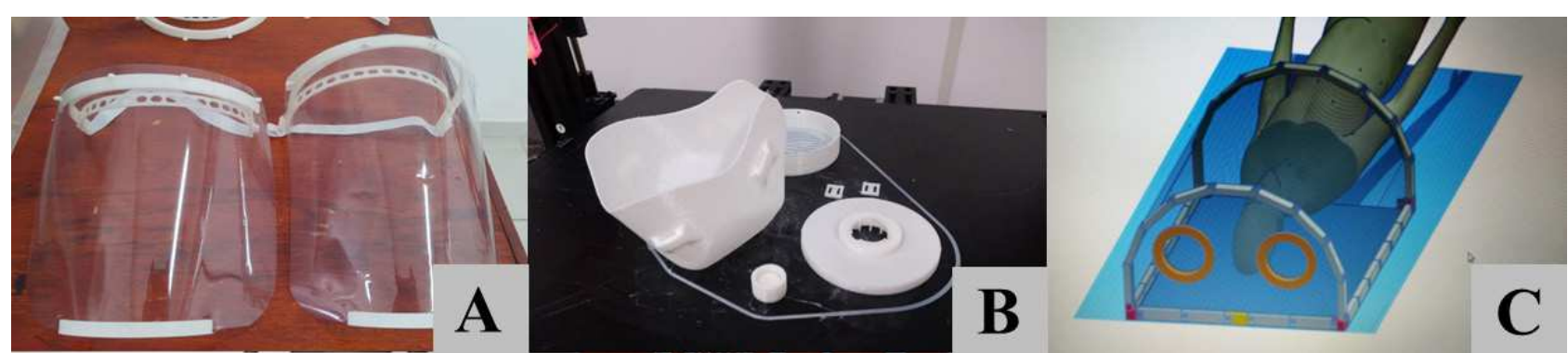

Fonte: UFAM (2020), Unila (2020) e UFJF (2020)

O protetor facial tipo "Face Shield" (Figura 2.A) é o modelo que tem sido mais produzido pelas instituições. Até o momento, 88 das 89 instituições encontradas informaram sua produção. Os protetores faciais são complementares à proteção individual, baseados no modelo desenvolvido pela Prusa Research, com sede na República Tcheca, que começou a compartilhar o código-aberto, permitindo que qualquer pessoa com uma impressora 3D faça o download e possa utilizar seu design gratuito (PRUSA PRINTERS, 2020). Diante do cenário de pandemia, a Agência Nacional de Vigilância Sanitária (ANVISA) orientou que os protetores faciais deveriam ser produzidos com folhas de acetato de $0,5 \mathrm{~mm}$, com estruturas de sustentação impressas em 3D e elástico que fixa o equipamento à cabeça do usuário, de acordo com as especificidades e requisitos estabelecidos na Resolução n. 356, de 23 de março de 2020 (ANVISA, 2020).

Vale lembrar que, além da escassez dos EPIs, as máscaras do modelo N-95 podem não ser suficientes para evitar a contaminação por partículas de coronavírus no ar, tanto pelo tamanho das partículas quanto por não proteger a região dos olhos, por isso, uma alternativa identificada para aumentar a proteção dos profissionais da saúde e também para aumentar a vida útil da máscara modelo N-95 e da máscara cirúrgica é o protetor facial, que se constitui em uma excelente alternativa (MORI, 2020; BRASIL, 2020b).

Até o momento das buscas realizadas, somente a Universidade Federal de Juiz de Fora (UFJF) não estava produzindo os protetores faciais, entretanto, é a única instituição que produzia o protótipo da cúpula em formato semicilíndrico (Figura 2.C) por meio do trabalho dos professores do Departamento de Física, do Instituto de Ciências Exatas (ICE), Rodrigo Alves Dias e José Paulo Mendonça de Furtado. A ideia da cúpula é permitir que as equipes médicas durante o tratamento de pacientes de novo coronavírus tenham acesso com as mãos de um lado e com aparelhos do outro, sendo essencial durante o processo de intubação traqueal de pacientes com COVID-19. Contudo, salienta-se que ainda é um protótipo e que precisa ser testado pelos profissionais da área. Tem-se o intuito de testar os primeiros protótipos no Hospital Universitário local, intencionando disponibilizar o projeto de montagem para todos os interessados em parcerias por todos o território nacional (UFJF, 2020).

Com relação à produção de protótipos similares às máscaras modelo N-95 em 3D (Figura 2.B), essas vêm sendo produzidas pela Universidade Federal da Integração Latino-Americana (UNILA), pela Universidade Federal do Maranhão (UFMA) e pelo Instituto Federal do Rio de Janeiro (IFRJ). Essas instituições federais uniram esforços para a confecção de máscaras que, posteriormente, poderão ser distribuídas aos hospitais locais. 
A Unila, por meio do projeto "Fabricação e impressão 3D de peças de equipamentos e EPIs para as ações de combate à Covid-19", produziu três modelos diferentes, similares às Máscaras modelo N-95 para uma testagem mais ampla no hospital municipal local, de modo que, inicialmente, fossem avaliados quais modelo, tipo de filtro e material - flexível ou rígido seriam mais aceitos para uso dos profissionais. Ressalta-se, ainda, que todo esse empenho tem sido subsidiado por doações e parcerias e que a instituição também está buscando recursos por meio de editais específicos para ações de combate à COVID-19, publicados pelo MEC e por agências de fomento. Recentemente, a Unila publicou edital para empresas, instituições e pessoas físicas interessadas em fazer doações de materiais para essa ação e outras que estão sendo desenvolvidas (UNILA, 2020).

Com relação à UFMA, a Superintendência de Infraestrutura (SINFRA), o Departamento de Desenho e Tecnologia (DEDET) e o Laboratório de Prototipagem e Design (intitulado como Fabrique) da UFAM, em parceria com a Secretaria Estadual da Ciência, Tecnologia e Inovação (SECTI), trabalharam tanto na confecção de protetores faciais como de máscaras modelo N-95, por meio do processo de termoformação com impressoras 3D. A confecção das máscaras modelo N-95 em 3D recebeu o apoio de profissionais da Sinfra, além de estudantes do Bacharelado em Ciência e Tecnologia (BICT) e do Programa de Pós-Graduação em Engenharia de Eletricidade (PPGEE) da universidade (UFMA, 2020).

No caso do IFRJ, a atuação foi realizada em duas frentes de trabalho, com professores dos campi Mesquita e Volta Redonda. O projeto consistiu na impressão em 3D de protótipo técnico das máscaras respiradores modelo N-95; impressão em 3D do suporte para os escudos faciais; compra e colocação dos filtros PFF2 nas máscaras; compra, preparação e colocação das folhas de acetato nos escudos; e compra e colocação de elásticos nos equipamentos. O IFRJ conta com o apoio institucional da Pró-Reitoria de Pesquisa, Inovação e Pós-Graduação (PROPPI) e da Agência de Inovação para a estruturação do programa de incentivo financeiro que está em construção. Atualmente, estão sendo recebidas doações por uma ação colaborativa com a empresa Start Up Inova Soluções Científicas e com suporte técnico de pesquisadores do Instituto Nacional de Tecnologia (IFRJ, 2020).

De acordo com o presente estudo, observou-se que, em tempos de pandemia, a solidariedade se alia à competência técnica e, com isso, a produção de EPIs tem sido ampliada $e$ debatida. Universidades e Institutos Federais do Brasil mobilizaram seus centros de pesquisas para ajudar no combate ao vírus Sars-CoV-2, em diversas frentes, principalmente na produção dos EPIs. Diante do exposto, houve a divulgação e a promoção dessas investigações em vários meios de comunicação, o que gerou um movimento cultural crescente dentro da comunidade de impressão 3D com o intuito de experimentar novos projetos de EPIs e distribuí-los abertamente por meio de projetos de "código-aberto" (JONES, 2020).

As ações de enfrentamento à COVID-19 distribuídas por todo o país, utilizando a impressão 3D, estão sendo coordenadas por professores, diretores e coordenadores dos laboratórios das próprias instituições, e o trabalho tem sido realizado por voluntários, que incluem a comunidade docente e discente, servidores técnicos, além do apoio de profissionais locais engajados na causa. Nesse sentido, o financiamento para custeio dessas ações tem sido proveniente de doações e de parcerias, subsidiadas por empresas privadas, laboratórios locais, secretarias estaduais ou municipais, vaquinhas on-line e editais de fomento internos lançados pelos próprios institutos e pelas universidades no apoio a ações de combate à COVID-19 (UFRB, 2020; UFAM, 2020). 
Um dos principais projetos e exemplo de parceria que vem produzindo o modelo Face Shield é o projeto Face Shield for Life 3D, que é formado por pesquisadores, de forma colaborativa e voluntária, da Universidade Federal do Recôncavo da Bahia (UFRB), da Escola Bahiana de Medicina e Saúde Pública (BAHIANA), da Universidade do Estado da Bahia (UNEB), da Universidade Federal do Oeste da Bahia (UFOB), do Centro Juvenil de Ciência e Cultura (CJCC), do Instituto Federal da Bahia (IFBA), da Secretaria de Ciência, Tecnologia e Inovação do Estado da Bahia (SECTI-BA), do Instituto PEPO, da Universidade Estadual de Feira de Santana (UEFS) e do Mdwood Eextrusion e Injeplast News, que, juntos, possuem a intenção de fornecer gratuitamente os EPIs fabricados em impressoras 3D para os profissionais de saúde da rede pública do Estado da Bahia (UFRB, 2020; JORGE et al., 2020).

No que se refere ao espaço de confecção dos equipamentos em geral, têm sido utilizados laboratórios próprios ou provenientes das parcerias, as quais atuam também cedendo em muitas das vezes algumas Impressoras 3D. Cabe destacar, também, que a equipe multiprofissional atuante frente aos protótipos é constituída por engenheiros, médicos, biólogos, pesquisadores, professores, estudantes, pessoas que possuem impressoras 3D, hospitais parceiros, que executam diferentes funções, como os testes de segurança, relatórios adicionais, ajustes e simplificação no modelo, tornando mais rápida e barata a produção, sem deixar de lado a segurança que todo equipamento médico necessita, com o propósito de avaliar e de aperfeiçoar os produtos, além de verificar o conforto, mesmo nesse momento de urgência (JONES, 2020; UNILA, 2020; UFAM, 2020). Por fim, com relação ao destino dos protótipos confeccionados, eles têm sido doados ao sistema público de saúde, como hospitais, unidades de saúde, aos profissionais da segurança pública e às unidades de pronto atendimento (JORGE et al., 2020; UFAM, 2020).

Um fator facilitador para a impressão das estruturas em 3D é o uso de materiais necessários, já que, assim, podem ser usados diversos polímeros, como o policarbonato, poliéster, cloreto de polivinil, entre outros. Esses biomateriais são transparentes, leves e oferecem alta clareza óptica, além de serem acessíveis e de baixo custo. Suas funcionalidades são destacadas por serem reutilizáveis, ajustáveis, por prevenir o contágio do vírus, de bactérias e de outras contaminações, pois evitam o contato com gotículas, salivas e fluidos nasais. Ademais, o polímero transparente permite ser facilmente higienizado e sua desinfecção é propiciada pela aplicação de álcool $70 \%$, hipoclorito de sódio ou outro desinfetante recomendado pelo fabricante ou pela Comissão de Controle de Infecção Hospitalar. Os materiais da impressão 3D também se destacam na produção de designs de peças personalizadas, como a vedação personalizada para melhorar o conforto e o ajuste da máscara similar ao modelo N-95 (UNILA, 2020), além de poder personalizar as características acerca das dimensões, deixando-a mais leve e confortável para o uso de longas horas de trabalho (PRUSA PRINTERS, 2020). Segundo Banerjee et al. (2019), isso é possível a partir da extrusão de materiais específicos que é usada para projetar um material elastomérico termoplástico imprimível em 3D com uma mistura de polipropileno (PP) e Estireno-Etileno-Butileno-Estireno (SEBS). Essa mistura fornece melhor capacidade de impressão no que se refere à flexibilidade e à elasticidade do material para modelo 3D para máscaras similares ao modelo N-95 e aos protetores faciais, resultando em equipamentos ajustáveis, personalizados, obtendo precisão, além do baixo custo.

Tendo em vista a escassez dos EPIs, as comunidades de impressão 3D se tornaram uma grande força motriz no esforço de produzi-los para aqueles que estão na linha de frente. Os EPIs em 3D exibem diversos fatores que contribuem e refletem na sua capacidade de resposta 
frente à pandemia atual e às futuras, por exemplo, a rápida disponibilidade e a produção dos protetores faciais, fato que só está sendo possível por causa do compartilhamento de informações e do software do protótipo com licenças de "código-aberto" ou "open source" (PRUSA PRINTERS, 2020; PROJETO HÍGIA, 2020). Nesse modelo, como já mencionado, os projetos têm sido desenvolvidos e compartilhados com licenças de código-aberto, o que permite que qualquer pessoa ou empresa possa acessar o protocolo de manufatura, desde que as informações de autoria inicial sejam mantidas, implementando a regra de citação científica no domínio, tendo em vista a proteção da propriedade intelectual (SEGNINI; ZAFALON, 2009). Com isso, usuários com conhecimentos e técnicas podem fazer adaptações no software de acordo com suas necessidades individuais, já que, para criar esses equipamentos, pode não se ter acesso ao mesmo equipamento no qual o software foi criado e, para tanto, seria necessário fazer pequenas modificações para otimizar determinados programas que possam ser executados em seus computadores e impressoras $3 \mathrm{D}$, além de possibilitar detectar e corrigir eventuais falhas do programa, bastando, para isso, obter uma impressora 3D, uma vez que dispensaram excepcional e temporariamente a autorização e a notificação à Anvisa, bem como outras autorizações sanitárias para a fabricação, a importação e a aquisição de dispositivos médicos identificados como prioritários para uso em serviços de saúde (ANVISA, 2020), o que viabiliza ainda mais sua escolha nesse momento de pandemia.

O uso da impressão 3D durante a pandemia da COVID-19, também, apresenta considerações e necessita de cuidados. A disponibilização dos hardwares de código-aberto e a produção desses protótipos geraram maior atenção da mídia, como a potencial alternativa à falta de EPIs aprovados para prevenção da transmissão da COVID-19. Órgãos internacionais se manifestaram, expondo a improbabilidade dos EPIs impressos em 3D fornecerem a mesma proteção que os EPIs aprovados por órgãos competentes (FAD, 2020; GALLAGHER, 2020). Foi criado, inclusive, um repositório on-line para protótipos de EPIs em 3D de código aberto para investigação e testagem, uma vez que são reconhecidas as limitações quanto a segurança, devido à heterogeneidade dos métodos de prototipagem rápida, mesmo com testes padrão (NIH, 2020; FAD, 2020).

A reprodutibilidade desses modelos é uma questão importante, embora o bloqueio mecânico de partículas possa ser replicado até certo ponto, os arquivos disponíveis fornecem apenas a forma virtual do modelo e é aberto, sendo passíveis de modificações e fabricação aditiva (GALLAGHER, 2020). Com pequenas diferenças nas variáveis de códigos entre duas impressoras, é possível até produzir dois modelos semelhantes, mas funcionalmente diferentes (GORDEEV; GALUSHKO; ANANIKOV, 2018). As propriedades eletrostáticas dos materiais impressos em 3D é outro ponto, já que pode variar a depender da composição do material, da porosidade e da estabilidade ambiental (KATKAR; TAFT; GRANT, 2018). Muitos filamentos retêm a umidade ambiente, o que pode representar um risco paradoxalmente aumentado de transmissão de vírus durante seu uso ou sua reutilização (JURISCHKA; DINTER; EFIMOVA, 2020).

Um ponto preocupante é que as autorizações de uso emergencial de materiais impressos em 3D quando são divulgadas, muitas vezes, não fornecem especificações de impressão para o desenvolvimento de protótipos (CLIFTON; DAMON; MARTIN, 2020), esse fator pode inviabilizar a produção e não garante a reprodutibilidade em vários tipos de impressoras. Essas informações são importantes para o fabricante e mantêm a qualidade, a segurança e a eficácia dos produtos fabricados. 
O baixo investimento no Brasil, com relação às políticas públicas para pesquisa e inovação frente à COVID-19 (HECK, 2020; DE NEGRI; KOELLER, 2020), gerou lacunas tecnológicas e desorganização das informações das iniciativas que estão sendo desenvolvidas em resposta a essa crise. Todos os esforços para suprir a demanda de EPIs também revelaram a fragilidade do país na sua capacidade produtiva e tecnológica. Para o enfrentamento da crise na pandemia, observou-se, também, o processo de desindustrialização e de engenharia reversa, copiando e fabricando o que já existia (SUZUKI; QUINTELLA, 2020).

A organização colaborativa entre instituições de ensino públicas, interligando pesquisadores, cientistas, laboratórios e hospitais, além de ter seu foco no combate à COVID-19, foi uma tentativa também de se superar as omissões em relação às políticas de apoio à ciência, à tecnologia e à inovação no Brasil (SANTOS; DA SILVA, 2019; PERES, 2020). As respostas para diversos problemas sociais dependem da ciência, da tecnologia e da capacidade de produção de conhecimento (DINIZ et al., 2020; TATUM et al.,2020).

\section{Considerações Finais}

Entre os EPIs mais produzidos pelos Institutos e Universidades Federais para o combate à COVID-19, estão os modelos de Protetores Faciais tipo "Face Shield", as máscaras similares ao modelo N-95 e a máscara com uma cúpula em formato semicilíndrico. As informações estão sendo compartilhadas por meio de licenças de "código-aberto".

A maior parte das instituições federais do Brasil atuou em ações de desenvolvimento $e$ de produção de EPIs durante o período de pandemia. O estudo identificou que essas instituições colocaram seus laboratórios e conhecimentos relativos às diferentes áreas à disposição da sociedade brasileira no combate à COVID-19, apresentando diversas possibilidades de intervenções, aliadas à inovação, ao baixo custo e à rápida produção, livre de patente e com avaliação científica, contribuindo na proteção e na segurança para os profissionais de saúde. Além disso, demonstra-se o engajamento multidisciplinar, o que proporcionou a criação de um movimento de suporte mútuo a grupos variados na criação e no desenvolvimento de projetos em suas regiões de atuação.

Observou-se a difusão de conhecimento qualificado à sociedade, de forma a pontuar que as instituições de ensino federais têm exercido seu papel fundamental de responsabilidade social, colocando em prática os três pilares que possuem, que é o ensino, a pesquisa e a extensão, auxiliando a comunidade nos problemas mais necessários e urgentes com ciência e tecnologia. Atrelado a esses fatores, o estudo também salienta a necessidade de expansão e de apoio das pesquisas voltadas para a eficácia desses equipamentos, de forma que se ofereça um sistema de retroalimentação interdisciplinar, de regulação de sua produção e de competência dos materiais utilizados, de forma a respeitar as orientações técnicas das autoridades de saúde.

\section{Perspectivas Futuras}

Associada às incertezas de sua repercussão, a rapidez com que se alastrou a COVID-19 trouxe consigo a evidência do despreparo social. A emergência da situação colocou em destaque os problemas relacionados ao desenvolvimento, à produção, à logística e à infraestrutura para 
atender a todos, e, no contexto em questão, revelou a indisponibilidade de EPIs aos múltiplos setores. A efetivação de medidas que possam suprir esses desafios de produção ainda perpassa por alguns problemas, trazendo a necessidade de tecer, como perspectivas futuras, a resolução dos impasses assumidos.

Para superar esses desafios, é necessário que haja investimentos em políticas públicas, em especial na área da pesquisa e de inovações tecnológicas, para o fomento dessa e de tantas outras ações no combate à COVID-19, atingindo, assim, todo potencial e conseguindo apresentar respostas efetivas.

A produção tecnológica, principalmente no aspecto da impressão 3D, tem cada vez mais trilhado um caminho de sua aplicação efetiva na sociedade. No entanto, percebe-se que mudanças são necessárias, muito embora elas já estejam ocorrendo, nas estratégias de transferências de tecnologia que as instituições de ensino federais possuem para, assim, direcionar tais estratégias para resolver os problemas sociais.

Espera-se que haja continuidade das múltiplas parcerias articuladas entres as diferentes áreas e setores sociais, como o Estado, as empresas privadas, as instituições de ensino e a sociedade civil. Deseja-se que essa articulação seja pensada e organizada para além de se obter velocidade de produção e baixo custo e que apresente segurança de uso e respeite os órgãos de segurança e de regulação.

\section{Referências}

ANAMT - ASSOCIAÇÃO NACIONAL DE MEDICINA DO TRABALHO. Enfermagem: Brasil tem 30 mortes de profissionais por coronavírus e mais de 4 mil afastados. Especial Coronavírus (COVID-19), 17 de abr. 2020. Disponível em: https://www.anamt.org.br/portal/2020/04/17/ enfermagem-brasil-tem-30-mortes-de-profissionais-por-coronavirus-e-mais-de-4-mil-afastados/. Acesso em: 7 maio 2020.

ANVISA - AGÊNCIA NACIONAL DE VIGILÂNCIA SANITÁRIA. Resolução - RDC n. 356, de 23 de março de 2020. Disponível em: http://www.in.gov.br/en/web/dou/-/resolucao-rdc-n356-de-23de-marco-de-2020-249317437. Acesso em: 24 ago. 2020

BANERJEE, S. S. et al. 3D-printable PP/SEBS thermoplastic elastomeric blends: Preparation and properties. Polymers, [S.l.], v. 11, n. 2, p. 347, 2019. Disponível: https://www.mdpi.com/20734360/11/2/347. Acesso em: 21 jun. 2020.

BRASIL. Ministério da Educação. Corona Vírus - Monitoramento nas Instituições de Ensino: ações de Enfrentamento na Rede Federal de Ensino. 2020a. Disponível em: http://portal.mec.gov.br/ coronavirus/. Acesso em: 12 maio 2020.

BRASIL. Ministério da Saúde. Secretaria de Vigilância em Saúde. Boletim Epidemiológico n. 7, Brasília, DF, Secretaria de Vigilância em Saúde SVS/MS-COE, 6 de abril de 2020 [2020b]. Disponível em: https://www.saude.gov.br/images/pdf/2020/April/06/2020-04-06-BE7-BoletimEspecial-do-COE-Atualizacao-da-Avaliacao-de-Risco.pdf. Acesso em: 21 jun. 2020.

BRASIL. Normas Regulamentadoras. NR 6 - Equipamento De Proteção Individual (206.000-0/ I0). Consolidação das Leis do Trabalho. 1978. Disponível em: http://www.trtsp.jus.br/geral/ tribunal2/LEGIS/CLT/NRs/NR_6.html. Acesso em: 5 maio 2020. 
CLIFTON, W.; DAMON, A.; MARTIN, A. K. Considerations and Cautions for ThreeDimensional-Printed Personal Protective Equipment in the COVID-19 Crisis. 3D Printing and Additive Manufacturing, 2020. Disponível em: https:/www.liebertpub.com/doi/ full/10.1089/3DP.2020.0101\#pane-pcw-references. Acesso em: 21 jun. 2020.

DE NEGRI, F.; KOELLER, P. Nota Técnica n. 64 (Diset): políticas públicas para pesquisa e inovação em face da crise da Covid-19. 2020. Diretoria de Estudos e Políticas Setoriais de Inovação e Infraestrutura. Disponível em: http://repositorio.ipea.gov.br/handle/11058/10034. Acesso em: 21 ago. 2020.

DINIZ, M. C. et al. Crise global coronavírus: monitoramento e impactos. Cadernos de Prospecção, Salvador, v. 13, n. 2, Especial COVID-19, p. 359-377, 2020. Disponível em: https://portalseer.ufba. br/index.php/nit/article/view/35937/20932. Acesso em: 23 ago. 2020.

FAD - FOOD AND DRUG ADMINISTRATION. 3D Printing of Medical Devices, Accessories, Components, and Parts During the COVID-19 Pandemic. Food and Drug Administration, 2020. Disponível em: https://www.fda.gov/medical-devices/3d-printing-medical-devices/faqs-3dprinting-medical-devices-accessories-components-and-parts-during-covid-19-pandemic. Acesso em: 21 jun. 2020.

GALLAGHER, M. B. 3 Questions: The risks of using 3D printing to make personal protective equipment. MIT News, [S.l.], 2020. Disponível em: http://news.mit.edu/2020/3q-risks-using-3dprinting-make-personal-protective-equipment-0326. Acesso em: 15 maio 2020.

GORDEEV, E. G.; GALUSHKO, A. S.; ANANIKOV, V. P. Improvement of quality of 3D printed objects by elimination of microscopic structural defects in fused deposition modeling. PLoS One, [S.l.], p. 13:e0198370, 2018. Disponível em: https://journals.plos.org/plosone/article?id=10.1371/ journal.pone.0198370. Acesso em: 25 jun. 2020.

HECK, Júlio Xandro. Inovação e Propriedade Intelectual no Brasil dos tempos da Covid-19. Cadernos de Prospecção, Salvador, v. 13, n. 4, p. 905, 2020.

IFRJ - INSTITUTO FEDERAL DE EDUCAÇÃO, CIÊNCIA E TECNOLOGIA DO RIO DE JANEIRO (IFRJ). IFRJ produz Equipamentos de Proteção Individual (EPI) por impressão 3D. 2020. Disponível em: https://portal.ifrj.edu.br/ifrj-produz-equipamentos-protecao-individual-epiimpressao-3d. Acesso em: 24 maio 2020.

JONES, J. Universidades se mobilizam para produzir EPIs. Pesquisa FAPESB. 2020. Disponível em: https://revistapesquisa.fapesp.br/universidades-se-mobilizam-para-produzir-epis/. Acesso em: 21 maio 2020.

JORGE, E. F. et al. Face Shield for Life 3D: produção colaborativa, usando a comunidade de makers, dos protetores faciais padrão RC3 para os profissionais de saúde em Salvador. Cadernos de Prospecção, Salvador, v. 13, n. 2, Especial COVID-19, p. 513-525, abr. 2020.

JURISCHKA, C.; DINTER, F.; EFIMOVA, A. et al. An explorative study of polymers for 3D printing of bioanalytical test systems. Clinical Hemorheology and Microcirculation, [S.l.], v. 75, n. 1, p. 57-84, 2020. Disponível em: https://content.iospress.com/articles/clinical-hemorheology-andmicrocirculation/ch190713. Acesso em: 25 jun. 2020.

KAMPF, G. et al. Persistence of coronaviruses on inanimate surfaces and its inactivation with biocidal agents. Journal of Hospital Infection, [S.I.], v. 104, n. 3, p. 246-251, 2020.

KATKAR, R. A.; TAFT, R. M.; GRANT, G. T.; 3D volume rendering and 3D printing (additive 
manufacturing). Dental Clinics of North America, [S.1.], v. 62. n. 3, p. 393-402, 2018. Disponível em: https://www.dental.theclinics.com/article/S0011-8532(18)30018-1/abstract. Acesso em: 25 jun. 2020.

LOCKHART, S. L. et al. Personal protective equipment (PPE) for both anesthesiologists and other airway managers: principles and practice during the COVID-19 pandemic. Canadian Journal of Anesthesia/Journal canadien d'anesthésie, [S.I.], p. 1-11, 2020. Disponível em: https://www. ncbi.nlm.nih.gov/pmc/articles/PMC7178924/. Acesso em: 21 maio 2020.

MATOZINHOS, I. P. et al. Impressão 3D: inovações no campo da medicina. Revista Interdisciplinar Ciências Médicas, MG, [S.l.], v. 1, n 1, p.143-162, 2017.

\section{MOOCK, M.; MELLO, P. M. V. de C. Pandemia COVID-19. Revista Brasileira de Terapia} Intensiva, [S.l.], v. 32, n. 1, p. 1, 2020.

MORI, L. Coronavírus: tudo o que você precisa saber sobre o uso ou não de máscara. BBC News Brasil, São Paulo, 26 mar. 2020. Disponível em: https://www.bbc.com/portuguese/brasil-52030633. Acesso em: 20 maio 2020.

NIH - NATIONAL INSTITUTE OF HEALTH 3D Print Exchange. COVID-19 Supply Chain Response. NIH 3D Print Exchange National Institutes of Health. [2020]. Disponível em: https://3dprint.nih. gov/collections/covid-19-response. Acesso em: 15 maio 2020.

OPAS - ORGANIZAÇÃO PAN-AMERICANA DE SAÚDE ([Brasil)]. Folha informativa - COVID-19 Escritório da OPAS e da OMS no Brasil. (Doença causada pelo novo coronavírus). Representação da OPAS no Brasil, Brasília, DF, 26 de abril de 2020. Disponível em: https:/www.paho.org/bra/ covid19/. Acesso em: 15 maio 2020.

PERES, A. C. À beira de um apagão: cortes de verbas para ciência e tecnologia fazem cientistas deixarem o Brasil. Escola Nacional de Saúde Pública Sergio Arouca/Fiocruz. Rio de Janeiro, 25 de junho de 2020. Disponível em: http://www.ensp.fiocruz.br/portal-ensp/informe/site/materia/ detalhe/46875. Acesso em: 25 jun. 2020.

\section{PROJETO HÍGIA. Protetores Faciais por impressão 3D para Hospitais em combate à}

COVID-19. [2020]. Disponível em: https://www.projetohigia.com.br/. Acesso em: 24 ago. 2020.

PRUSA PRINTERS. Prusa Face Shield. República Tcheca. 2020. Disponível em: https://www. prusaprinters.org/prints/25857-prusa-protective-face-shield-rc2. Acesso em: 5 abril 2020.

RAMSEY, L. Hospitals could be overwhelmed with patients and run out of beds and ventilators as the coronavirus pushes the US healthcare system to its limits. Business Insider India. 2020. Disponivel em: https://www.businessinsider.in/science/news/hospitals-could-be-overwhelmed-withpatients-and-run-out-of-beds-and-ventilators-as-the-coronavirus-pushes-the-us-healthcare-system-toits-limits/articleshow/74579074.cms. Acesso em: 15 maio 2020.

SANTOS, M. H. S.; DA SILVA, R. B. P. A crise da ciência, tecnologia e inovação no Brasil pós 2016. Revista Políticas Públicas \& Cidades, [S.I.], v. 8, n. 2, p. 66-77, 2019.

SEGNINI, R. C.; ZAFALON, Z. R. Copyright e copyleft: estudo dos direitos de acesso à informação $e$ do direito do leitor. In: XVI SEMINÁRIO NACIONAL DE BIBLIOTECAS UNIVERSITÁRIAS. UFRJ, 2010. Anais Eletrônicos. Rio de Janeiro: UFRJ, 2010. Disponível em: http://eprints.rclis.org/ handle/10760/15355. Acesso em: $1^{\circ}$ set. 2020. 
SUZUKI, H.; QUINTELLA, C. M. Reflexões sobre o grau de (des)preparo tecnológico do país evidenciadas pela COVID-19. E o que nós (autores e leitores da revista Cadernos de Prospecção) temos a ver com isso? Cadernos de Prospecção, Salvador, v. 13, n. 2, Especial COVID-19, p. 311, 2020.

TANNE, J. H. et al. Covid-19: how doctors and healthcare systems are tackling coronavirus worldwide. The BMJ., [S.l.], mar. 2020. Disponível em: https://www.bmj.com/content/368/bmj. m1090. Acesso em: 7 maio 2020.

TATUM, C. T. S. et al. Coronavírus no Processo de Impactação Científica Global. Cadernos de Prospecção, Salvador, v. 13, n. 2, Especial COVID-19, p. 494-512, 2020. Disponível em: https:// portalseer.ufba.br/index.php/nit/article/view/36136. Acesso em: 23 ago. 2020.

UFAM - UNIVERSIDADE FEDERAL DO AMAZONAS. Impressão 3D: UFAM inicia a produção de Equipamentos de Proteção Individual em Itacoatiara. 2020. Disponível em: https://www.ufam.edu. br/noticias-coronavirus/1250-impressao-3d-ufam-inicia-a-producao-de-equipamentos-de-protecaoindividual-em-itacoatiara.html. Acesso em: 15 maio 2020.

UFJF - UNIVERSIDADE FEDERAL DE JUIZ DE FORA. Pesquisadores da UFJF utilizam impressora 3D para desenvolver protótipo de EPI. 2020. Disponível em: https://www2.ufjf.br/ noticias/2020/03/25/pesquisadores-da-ufjf-utilizam-impressora-3d-para-desenvolver-prototipode-epi/. Acesso em: 24 maio 2020.

UFMA - UNIVERSIDADE FEDERAL DO MARANHÃO. Setores da UFMA unem esforços para confecção de máscaras e protetores faciais para o HU-UFMA. 2020. Disponível em: https:// portais.ufma.br/PortalUfma/paginas/noticias/noticia.jsf?id=56019. Acesso em: 24 maio 2020.

UFRB - UNIVERSIDADE FEDERAL DO RECÔNCAVO DA BAHIA. UFRB produz máscara de proteção 3D contra CoVid-19 para profissionais de saúde. 2020. Disponível em: https:// ufrb.edu.br/portal/noticias/5788-ufrb-produz-mascara-de-protecao-3d-contra-covid-19-paraprofissionais-de-saude. Acesso em: 24 maio 2020.

UNILA - UNIVERSIDADE FEDERAL DA INTEGRAÇÃO LATINO-AMERICANA. UNILA produz máscaras em impressoras 3D para uso de profissionais do Hospital Municipal. 2020. Disponível em: https://portal.unila.edu.br/noticias/unila-usa-impressora-3d-para-produzirmascaras-reutilizaveis. Acesso em: 24 maio 2020.

VAN DOREMALEN, N. et al. Aerosol and surface stability of SARS-CoV-2 as compared with SARSCoV-1. New England Journal of Medicine, [S.1.], v. 382, n.16, p. 1.564-1.567, abril de 2020.

WHO - WORLD HEALTH ORGANIZATION. WHO Director-General's opening remarks at the media briefing on COVID-19, 11 March 2020. World Health Organization. 11 de mar. de 2020.

\title{
Sobre os Autores
}

\author{
Amanda Ferreira dos Santos \\ E-mail: amanda.santos@csc.ufsb.edu.br \\ Graduanda em Medicina pela Universidade Federal do Sul da Bahia (UFSB). \\ Endereço profissional: Universidade Federal do Sul da Bahia, Rodovia de Acesso para Itabuna, km 39, Ferradas, \\ Itabuna, BA. CEP: 45613-204.
}




\section{Iulas de Souza Ramos}

E-mail: iulas.ramos@cpf.ufsb.edu.br Graduando em Interdisciplinar em Saúde pela Universidade Federal do Sul da Bahia (UFSB).

Endereço profissional: Universidade Federal do Sul da Bahia, Rodovia de Acesso para Itabuna, km 39, Ferradas, Itabuna, BA. CEP: 45613-204.

\section{Ana Clara Silva dos Santos}

E-mail: ana.santos@cpf.ufsb.edu.br Graduanda em Interdisciplinar em Saúde pela Universidade Federal do Sul da Bahia (UFSB).

Endereço profissional: Universidade Federal do Sul da Bahia, Rodovia de Acesso para Itabuna, km 39, Ferradas, Itabuna, BA. CEP: 45613-204.

\section{Grasiely Faccin Borges}

E-mail: grasiely.borges@gmail.com

Doutorado em Ciências do Desporto pela Faculdade de Ciências do Desporto e Educação Física (FCDEF-UC) pela Universidade de Coimbra, Portugal, em 2013.

Endereço profissional: Universidade Federal do Sul da Bahia, Rodovia de Acesso para Itabuna, km 39, Ferradas, Itabuna, BA. CEP: 45613-204.

\section{Ita de Oliveira e Silva}

E-mail:itabio@hotmail.com

Doutorado em Biologia Animal pela Universidade de Brasília em 2008.

Endereço profissional: Universidade Federal do Sul da Bahia, Rodovia de Acesso para Itabuna, km 39, Ferradas, Itabuna, BA. CEP: 45613-204.

\section{Jane Mary de Medeiros Guimarães}

E-mail: janemg@gmail.com

Doutorado em Saúde coletiva pela Instituto de Saúde Coletiva (ISC/UFBA) em 2014.

Endereço profissional: Universidade Federal do Sul da Bahia, Rodovia de Acesso para Itabuna, km 39, Ferradas, Itabuna, BA. CEP: 45613-204. 